AperTO - Archivio Istituzionale Open Access dell'Università di Torino

\title{
Exploring the Solar Wind from Its Source on the Corona into the Inner Heliosphere during the First Solar Orbiter-Parker Solar Probe Quadrature
}

\section{This is the author's manuscript}

Original Citation:

\section{Availability:}

This version is available http://hdl.handle.net/2318/1828388

since 2021-12-27T11:28:18Z

Published version:

DOI:10.3847/2041-8213/ac282f

Terms of use:

Open Access

Anyone can freely access the full text of works made available as "Open Access". Works made available under a Creative Commons license can be used according to the terms and conditions of said license. Use of all other works requires consent of the right holder (author or publisher) if not exempted from copyright protection by the applicable law. 


\section{Exploring the Solar Wind from Its Source on the Corona into the Inner Heliosphere during the First Solar Orbiter-Parker Solar Probe Quadrature}

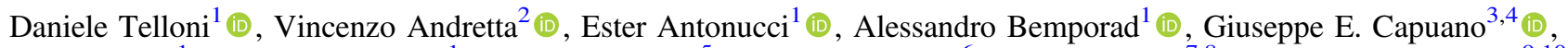

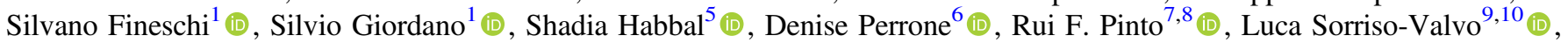

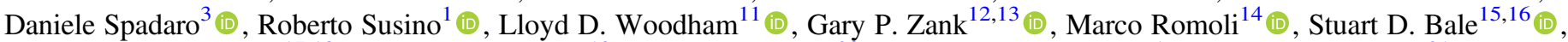

Justin C. Kasper ${ }^{17,18}$ (1) , Frédéric Auchère ${ }^{19}$ (D) , Roberto Bruno $^{20}$ (1) , Gerardo Capobianco $^{1}$ (1) , Anthony W. Case ${ }^{21}$ (1), Chiara Casini 22,23 (10), Marta Casti ${ }^{24}$ (1), Paolo Chioetto ${ }^{22}$ (1), Alain J. Corso ${ }^{22}$ (1), Vania Da Deppo ${ }^{22}$ (1), Yara De Leo ${ }^{4,25}$ (1),

Thierry Dudok de $\mathrm{Wit}^{26}$ (1) , Federica Frassati ${ }^{1}$ (1) , Fabio Frassetto ${ }^{22}$ (1) , Keith Goetz $^{27}$ (1), Salvo L. Guglielmino ${ }^{3}$ (1),

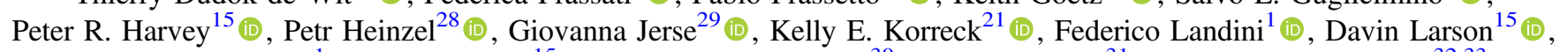
Alessandro Liberatore ${ }^{1}$ (1) , Roberto Livi ${ }^{15}$ (1), Robert J. MacDowall ${ }^{30}$ (1), Enrico Magli ${ }^{31}$ (1), David M. Malaspina ${ }^{32,33}$ (1),

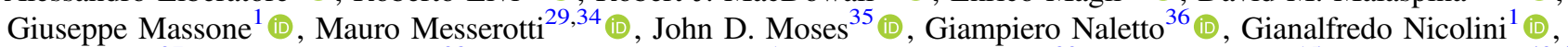

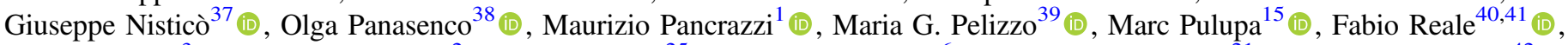

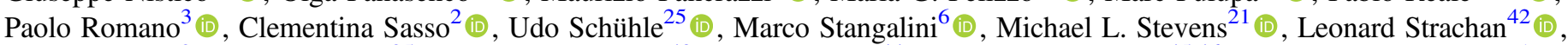

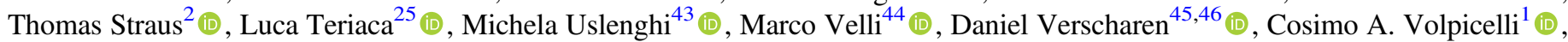
Phyllis Whittlesey ${ }^{15}$ (ii), Luca Zangrilli ${ }^{1}$ (i) , Gaetano Zimbardo ${ }^{37}$ (i), and Paola Zuppella ${ }^{22}$ (i)

${ }^{1}$ National Institute for Astrophysics, Astrophysical Observatory of Torino, Via Osservatorio 20, I-10025 Pino Torinese, Italy; daniele.telloni@inaf.it

${ }^{2}$ National Institute for Astrophysics, Astronomical Observatory of Capodimonte, Salita Moiariello 16, I-80131 Napoli, Italy

${ }^{3}$ National Institute for Astrophysics, Astrophysical Observatory of Catania, Via Santa Sofia 78, I-95123 Catania, Italy

${ }^{4}$ University of Catania, Department of Physics and Astronomy, Via Santa Sofia 64, I-95123 Catania, Italy

${ }^{5}$ Institute for Astronomy, University of Hawaii, Honolulu, HI 96822, USA

${ }^{6}$ Italian Space Agency, Via del Politecnico snc, I-00133 Roma, Italy

${ }^{7}$ Département dAstrophysique, Astrophysique Instrumentation Modélisation, Commissariat à l'Énergie Atomique et aux Énergies Alternatives, Institut de Recherche sur les lois Fondamentales de l'Univers, Centre National de la Recherche Scientifique, Institut National des Sciences de l’Univers, Université Paris-Saclay, Université de Paris, Orme des Merisiers Bât 709, F-91191 Gif-sur-Yvette, France

${ }^{8}$ Institut de Recherche en Astrophysique et Planétologie, Université de Toulouse, Université fe Paul Sabatier, Midi-Pyrénées Observatory, Centre National de la Recherche Scientifique, 9 Avenue du Colonel Roche, F-31028 Toulouse, France

${ }^{9}$ Swedish Institute of Space Physics, Angström Laboratory, Lägerhyddsvägen 1, SE-751 21 Uppsala, Sweden

${ }^{10}$ National Research Council, Institute for the Science and Technology of Plasmas, Via Amendola 122/D, I-70126 Bari, Italy

${ }^{11}$ Department of Physics, Imperial College London, London SW7 2AZ, UK

${ }^{12}$ Center for Space Plasma and Aeronomic Research, University of Alabama in Huntsville, Huntsville, AL 35805, USA

${ }^{13}$ Department of Space Science, University of Alabama in Huntsville, Huntsville, AL 35805, USA

${ }^{14}$ University of Florence, Department of Physics and Astronomy, Via Giovanni Sansone 1, I-50019 Sesto Fiorentino, Italy

${ }^{15}$ Space Sciences Laboratory, University of California, Berkeley, CA 94720, USA

${ }^{6}$ Physics Department, University of California, Berkeley, CA 94720, USA

${ }^{17}$ BWX Technologies, Inc., Washington, DC 20002, USA

${ }^{18}$ Climate and Space Sciences and Engineering, University of Michigan, Ann Arbor, MI 48109, USA

${ }^{19}$ Université Paris-Saclay, Centre National de la Recherche Scientifique, Institut d'Astrophysique Spatiale, Rue Jean-Dominique Cassini, F-91440 Bures-sur-Yvette, France

${ }^{20}$ National Institute for Astrophysics, Institute for Space Astrophysics and Planetology, Via del Fosso del Cavaliere 100, I-00133 Roma, Italy

${ }^{21}$ Harvard-Smithsonian Center for Astrophysics, Cambridge, MA 02138, USA

${ }^{22}$ National Research Council, Institute for Photonics and Nanotechnologies, Via Trasea 7, I-35131 Padova, Italy

${ }^{24}$ The Catholic University of America at the National Aeronautics and Space Administration, Goddard Space Flight Center, Greenbelt, MD 20771, USA

${ }^{25}$ Max Planck Institute for Solar System Research, Justus-von-Liebig-Weg 3, D-37077 Göttingen, Germany

${ }^{26}$ Laboratoire de Physique et de Chimie de l'Environnement et de l'Espace, Center National de la Recherche Scientifique, 3A Avenue de la Recherche Scientifique, F-45071 Orléans, France

${ }^{27}$ School of Physics and Astronomy, University of Minnesota, Minneapolis, MN 55455, USA

${ }^{28}$ Czech Academy of Sciences, Astronomical Institute, Fričova 298, CZ-25165 Ondřejov, Czech Republic

${ }^{29}$ National Institute for Astrophysics, Astronomical Observatory of Trieste, Località Basovizza 302, I-34149 Trieste, Italy

${ }^{30}$ National Aeronautics and Space Administration, Goddard Space Flight Center, Greenbelt, MD 20771, USA

${ }^{31}$ Politecnico of Turin, Department of Electronics and Telecommunications, Corso Duca degli Abruzzi 24, I-10129 Torino, Italy

${ }_{32}$ Astrophysical and Planetary Sciences Department, University of Colorado, Boulder, CO 80309, USA

${ }^{33}$ Laboratory for Atmospheric and Space Physics, University of Colorado, Boulder, CO 80303, USA

${ }^{34}$ University of Trieste, Department of Physics, Via Alfonso Valerio 2, I-34127 Trieste, Italy

${ }^{35}$ National Aeronautics and Space Administration, Headquarters, Washington, DC 20546, USA

${ }^{36}$ University of Padua, Department of Physics and Astronomy, Via Francesco Marzolo 8, I-35131 Padova, Italy

${ }^{37}$ University of Calabria, Department of Physics, Ponte Pietro Bucci Cubo 31C, I-87036 Rende, Italy

${ }^{38}$ Advanced Heliophysics, Pasadena, CA 91106, USA

${ }^{39}$ National Research Council, Institute of Electronics, Information Engineering and Telecommunications, Via Gradenigo 6/B, I-35131 Padova, Italy

${ }^{40}$ University of Palermo, Department of Physics and Chemistry-Emilio Segrè, Piazza del Parlamento 1, I-90134 Palermo, Italy

${ }^{41}$ National Institute for Astrophysics, Astronomical Observatory of Palermo, Piazza del Parlamento 1, I-90134 Palermo, Italy

${ }^{43}$ National Institute for Astrophysics, Institute of Space Astrophysics and Cosmic Physics of Milan, Via Alfonso Corti 12, I-20133 Milano, Italy

${ }^{44}$ Earth, Planetary, and Space Sciences, University of California, Los Angeles, CA 90095, USA

${ }^{45}$ Mullard Space Science Laboratory, University College London, Holmbury St. Mary, Dorking RH5 6NT, UK

${ }^{46}$ Space Science Center, Institute for the Study of Earth, Oceans, and Space, University of New Hampshire, Durham, NH 03824, USA

Received 2021 August 15; revised 2021 September 15; accepted 2021 September 17; published 2021 October 7 


\begin{abstract}
This Letter addresses the first Solar Orbiter (SO)-Parker Solar Probe (PSP) quadrature, occurring on 2021 January 18 to investigate the evolution of solar wind from the extended corona to the inner heliosphere. Assuming ballistic propagation, the same plasma volume observed remotely in the corona at altitudes between 3.5 and 6.3 solar radii above the solar limb with the Metis coronagraph on SO can be tracked to PSP, orbiting at 0.1 au, thus allowing the local properties of the solar wind to be linked to the coronal source region from where it originated. Thanks to the close approach of PSP to the Sun and the simultaneous Metis observation of the solar corona, the flow-aligned magnetic field and the bulk kinetic energy flux density can be empirically inferred along the coronal current sheet with an unprecedented accuracy, allowing in particular estimation of the Alfvén radius at 8.7 solar radii during the time of this event. This is thus the very first study of the same solar wind plasma as it expands from the subAlfvénic solar corona to just above the Alfvén surface.
\end{abstract}

Unified Astronomy Thesaurus concepts: Magnetohydrodynamics (1964); Space plasmas (1544); Interplanetary turbulence (830); Solar corona (1483); Heliosphere (711); Solar wind (1534)

\section{Introduction}

Remote sensing observations are essential to study the global magnetic configuration of the solar corona and to explore the acceleration regions of the solar wind (Antonucci et al. 2020a). However, complications in integrating along the line of sight (LOS; e.g., Dolei et al. 2018) over an extended region of the corona (an optically thin plasma; e.g., Bradshaw \& Raymond 2013) and the low cadence measurements of coronagraphs make it difficult to investigate in detail local coronal heating, identify high-frequency waves of different kinds, or study turbulent properties of the coronal plasma as a magnetohydrodynamic fluid. On the other hand, in situ measurements provide direct physical parameters of the plasma at a single point in space and time, enabling the examination of the local mechanisms that control, e.g., energy dissipation in the solar wind (Bruno \& Carbone 2013; Verscharen et al. 2019). However, single-spacecraft in situ measurements alone cannot provide topological information about the magnetic field, and thus a three-dimensional view of the plasma surrounding the spacecraft. Therefore, the combination of remote sensing observations and in situ measurements provides an unequaled opportunity to connect solar wind source regions to the local properties of plasma and electromagnetic fields. This link works best for in situ measurements close to the Sun, where solar plasma is not yet fully reprocessed by streamstream interactions.

There are several possible orbital configurations between two or more spacecraft relative to the Sun that allow the study of the connection of the plasma observed in situ to its solar source. One interesting case is provided by "quadratures," i.e., when the angular separation of two spacecraft with the Sun as reference point is $90^{\circ}$. Important examples of this kind of quadrature are given by the SOlar and Heliospheric Observatory (SOHO; Domingo et al. 1995)-Sun-Ulysses (Wenzel et al.

1992) campaigns, when the same plasma remotely observed with SOHO was sampled in situ when it left the coronal source in the direction of Ulysses (Suess et al. 2000; Suess \& Poletto 2001; Poletto et al. 2002). The comparison of plasma properties in the corona and heliosphere was made possible thanks to the combined use of the Large Angle Spectroscopic COronagraph (LASCO; Brueckner et al. 1995) to understand the overall coronal configuration at the time of quadratures, and the UltraViolet Coronagraphic Spectrometer (UVCS; Kohl et al. 1995) to derive its physical characteristics, on SOHO, and the Solar Wind Ion Composition Spectrometer (SWICS; Gloeckler et al. 1992) to provide interplanetary data, on
Ulysses. Multiple quadratures (twice a year) during the SOHOUlysses era allowed the study of solar wind parameters, such as density, speed and temperature, and their evolution from the Sun to the heliosphere. Other quantities, such as ion abundances or the ionization state, which instead remain almost unchanged during solar wind expansion, have been used to conclusively link a plasma stream measured in situ with the coronal source as identified in coronagraphic images (Bemporad et al. 2003).

The launch of Parker Solar Probe (PSP; Fox et al. 2016) in 2018 August and Solar Orbiter (SO; Müller et al. 2020) in 2020 February, offers an exciting new era to explore the connection between the Sun and the heliosphere. On the one hand, PSP will be the first spacecraft to fly into the extended solar corona, a yet unexplored environment with in situ experiments. On the other hand, SO is the first spacecraft to carry both remote sensing and in situ instruments at distances as close as $0.28 \mathrm{au}$. It will leave the ecliptic plane, allowing for the first time observation of the solar poles, the regions where the fast solar wind originates (during solar minimum) and where magnetic fields cyclically reverse (Zouganelis et al. 2020). The importance of this new era for solar physics also lies in the synergy between these two missions (Velli et al. 2020), which will allow the tracing of the magnetic connectivity of transient outflows and the continuous solar wind to their solar sources. Particular attention has been paid to radial alignments between the two probes. Indeed, the first PSP-SO line-up occurred in 2020 September, allowing the radial evolution of solar wind turbulence between 0.1 and 1 au to be studied, i.e., from a highly Alfvénic and less developed turbulence state near the Sun to a fully developed and intermittent turbulence state close to the Earth (Telloni et al. 2021).

As far as it concerns quadrature configurations between the two spacecraft, with SO remotely observing the source region of the solar wind that is measured in situ with PSP, the first SO-Sun-PSP quadrature occurred on 2021 January 18. At that time, when traveling along its orbit very close to the Sun, PSP crossed the very inner heliosphere at a heliocentric distance just above 20 solar radii $\left(\mathrm{R}_{\odot}\right)$, while the coronagraph Metis (Antonucci et al. 2020b) on SO was observing the solar atmosphere in an annular field of view (FOV) extending from 3.5 to $6.3 R_{\odot}$ above the solar limb. It is thus the first time that an expanding coronal plasma, observed remotely with Metis, is almost immediately measured as it propagates outward with a suite of in situ instruments.

This Letter presents joint SO-PSP observations that describe the evolution of the pristine solar wind, not yet reprocessed by 

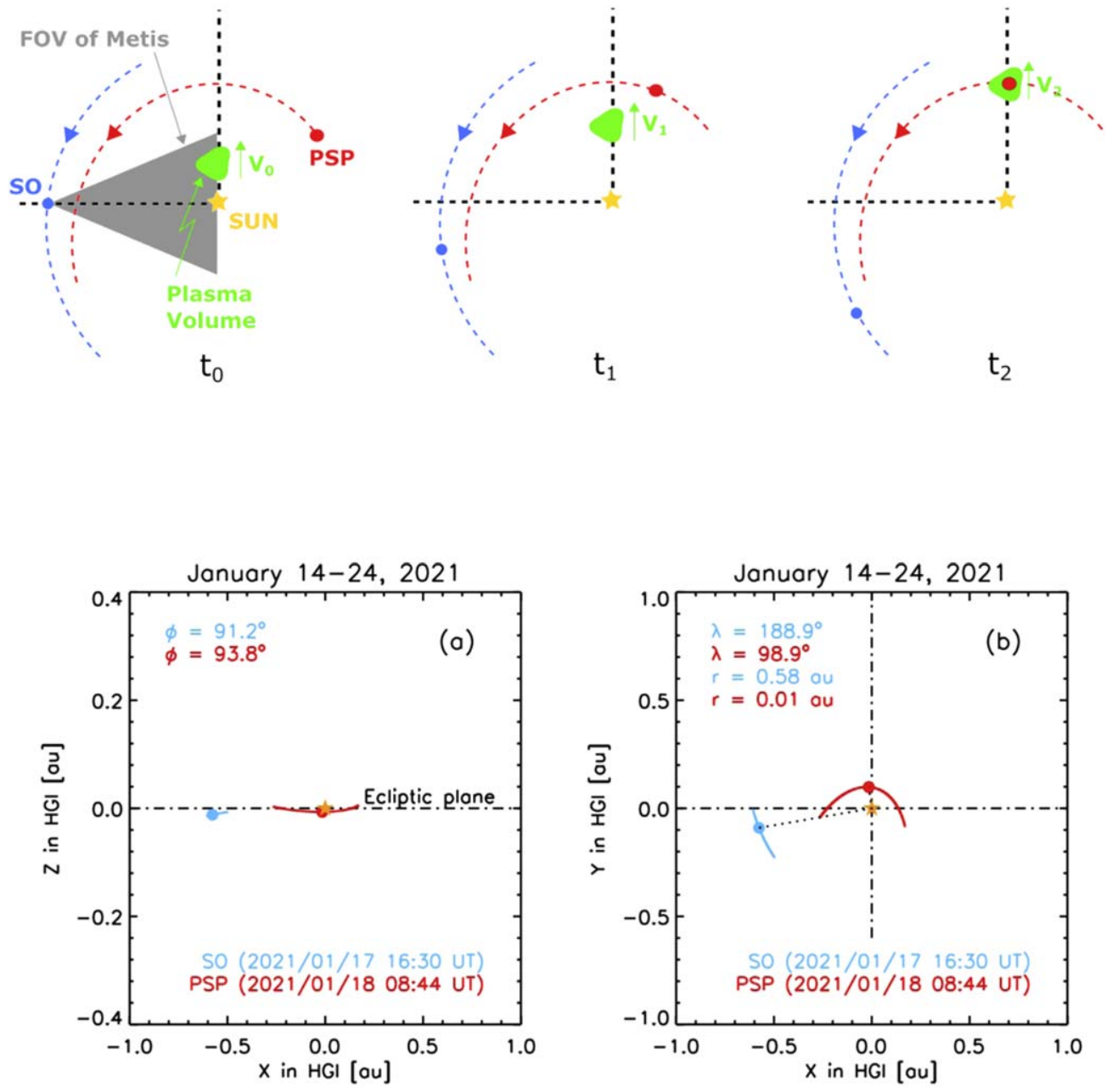

Figure 1. Top panel: cartoon showing how the same plasma volume observed remotely with SO/Metis can be measured locally with PSP during their quadrature. Bottom panels: SO (blue) and PSP (red) positions relative to the Sun (yellow star), in side (a) and top (b) views of the ecliptic plane in the HGI coordinate system, at the times (reported in the legends) of the observation of the same solar wind stream. The corresponding heliocentric distances $(r)$, and heliographic latitudes $(\phi)$ and longitudes $(\lambda)$ are also reported. The spacecraft trajectories from 2021 January 14-21, and their longitudinal separation of $90^{\circ}$ in the $X Y$ plane are shown as colorcoded solid and black dotted lines, respectively.

nonlinear interactions, from the corona into the very inner heliosphere, aiming to link the local properties of the plasma stream measured in situ with PSP with the coronal source from where it originated imaged by the $\mathrm{SO} /$ Metis coronagraph.

\section{Analysis and Results}

The SO-PSP orbital configuration offers the opportunity to track the same plasma volume as it expands from the extended corona to the inner heliosphere. Indeed, thanks to the continuous expansion of the solar corona, the two probes will at some point be located such that the plasma crossed by PSP, which is moving outward at a speed around $100-200 \mathrm{~km} \mathrm{~s}^{-1}$ on the solar equatorial plane, is the same plasma observed with Metis just a few hours earlier at a distance of 3.5-6.3 $R_{\odot}$. From the cartoon of Figure 1 (top panel), this condition requires that during Metis observations, PSP is not yet on the instantaneous plane of the sky (POS) of the coronagraph, so that PSP and the Metis-observed volume of plasma reach the same location at the same time.

On the basis of the spacecraft's ephemerides and (as shown below) of the solar wind speed at both coronal and heliospheric heights, this particular condition occurred when Metis was observing the solar corona on 2021 January 17 at 16: 30 UT $\left(t_{0}\right.$ in the cartoon of Figure 1). At that time PSP was about $30^{\circ}$ behind the instantaneous POS of Metis and it took $\sim 16.2 \mathrm{hr}$ to travel that longitudinal separation, thus reaching the Metis POS on 2021 January 18 at 08: 44 UT ( $t_{2}$ in the cartoon of Figure 1). This occurred when PSP was approaching perihelion at 0.1 au from the Sun and at a latitude of 3.8 below the ecliptic plane ( i.e., $93^{\circ} .8$ counterclockwise from the North Pole). As shown below (based on the expansion velocity inferred from Metis observations), the plasma observed (at $t_{0}$ ) with Metis at a distance of 3.5-6.3 $R_{\odot}$ took $16.3 \pm 1.1 \mathrm{hr}$ to travel the distance from PSP thus encountering it on its way outward. The corresponding SO and PSP locations are shown along their orbits in the $X Z$ and $X Y$ planes of the HelioGraphic Inertial (HGI) coordinate frame of Figures 1(a) and (b), respectively.

A time interval of about $2.5 \mathrm{hr}$ centered on $t_{2}$, comparable to the coronal plasma transit time through the Metis FOV (according to the speed of the outflowing plasma shown below) and resulting in a slight off-quadrature of $\pm 2^{\circ}$ longitude relative to the Metis POS, is thus identified in PSP data as corresponding to the same plasma volume observed remotely with Metis $16.3 \mathrm{hr}$ earlier. Some relevant parameters during this time period are displayed between the vertical dotted lines in Figure 2, which overall spans a time interval of 3.5 days. 


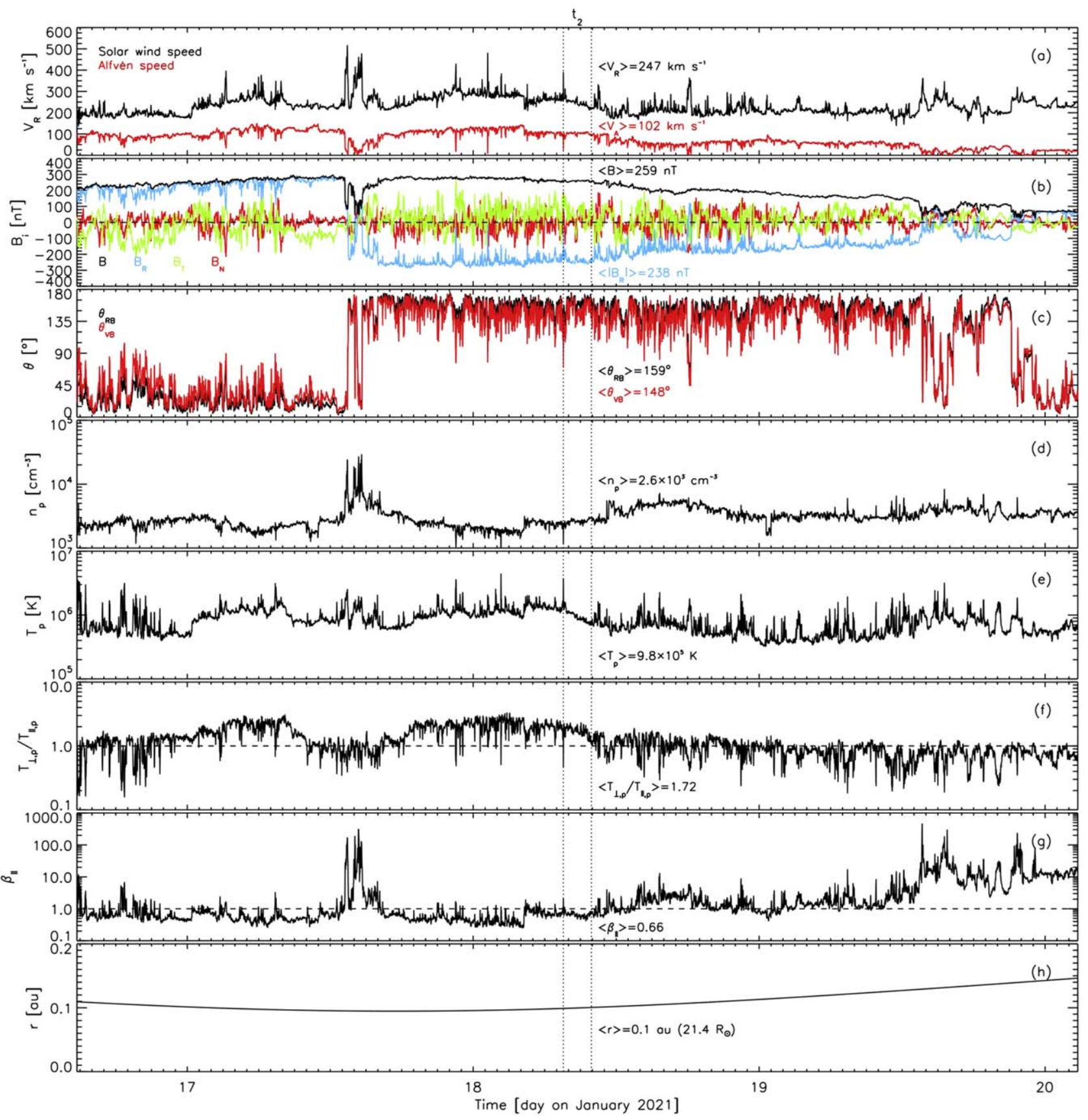

Figure 2. PSP solar wind parameters over the 3.5 day window centered on the $\sim 2.5 \mathrm{hr}$ long selected time interval (delimited by vertical dotted lines) around $t_{2}$ : solar wind radial speed $V_{R}$ and Alfvén speed $V_{A}$ (a), magnetic field vector $\boldsymbol{B}(\mathrm{b})$, angles $\theta_{\mathrm{RB}}$ and $\theta_{\mathrm{VB}}$ of $\boldsymbol{B}$ with the radial and velocity directions (c), proton number density $n_{p}(\mathrm{~d})$, temperature $T_{p}(\mathrm{e})$, temperature anisotropy $T_{\perp, p} / T_{\|, p}(\mathrm{f})$, and parallel plasma beta $\beta_{\|}(\mathrm{g})$, and radial distance $r(\mathrm{~h})$. The average values reported within each panel refer to the identified period.

Specifically, magnetic field and plasma measurements were acquired with the fluxgate magnetometer of the FIELDS suite (Bale et al. 2016) and with the SPAN-Ai top-hat electrostatic analyzer of the Solar Wind Electrons Alphas \& Protons suite (SWEAP; Kasper et al. 2016), respectively, and then merged at 1 minute resolution. As described by Woodham et al. (2021), the core proton velocity distributions have been fitted to a biMaxwellian function to obtain plasma moments (i.e., proton density, velocity, and temperature).

The solar wind radial speed $V_{R}$ and the Alfvén speed $V_{A}$ are displayed in Figure 2(a), showing that the plasma flow, despite being slow, is largely super-Alfvénic. Several spikes indicate bursts of accelerated wind. The magnetic field $\boldsymbol{B}$, in the inertial
Radial Tangential Normal (RTN) frame, and its angles with the radial $\left(\theta_{\mathrm{RB}}\right)$ and solar wind $\left(\theta_{\mathrm{VB}}\right)$ directions are shown in Figures 2(b) and (c), respectively. While the large-scale time profile of the magnetic field radial component is attributed to the expected $1 / r^{2}$ scaling, with $r$ the radial distance displayed in Figure 2(h), two sharp magnetic field $\sim 180^{\circ}$ rotations clearly indicate crossings of the heliospheric current sheet (HCS), so that during the interval under study the magnetic field is directed sunward. Several sharp rotations are present, coinciding with wind accelerations, although these do not reverse the field direction. In particular, there are no switchbacks (Bale et al. 2019) during the selected interval. The very small difference between $\theta_{\mathrm{RB}}$ and $\theta_{\mathrm{VB}}$ indicates that the solar 

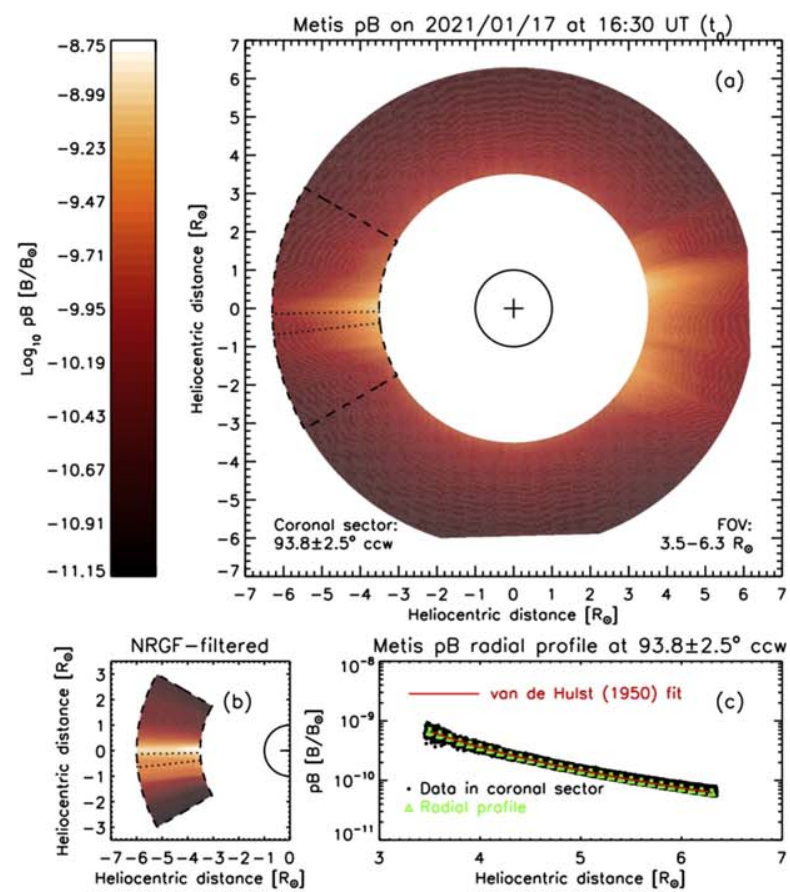

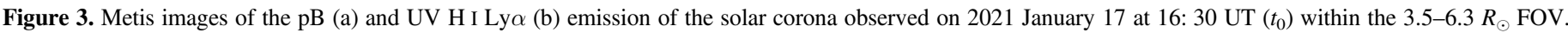

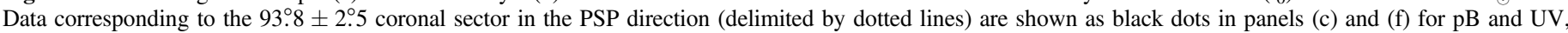

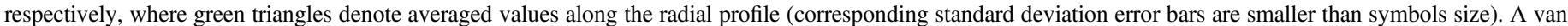

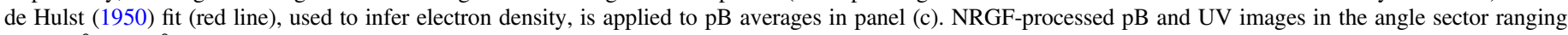
from $60^{\circ}$ to $120^{\circ}$ (indicated by dashed lines) are displayed in panels (b) and (e), respectively, to enhance coronal structures near the region under study.

wind velocity is nearly radial, thus supporting the assumption of a radially expanding coronal plasma underlying the ballistic assumption used in this work. Finally, Figures 2(d)-(g) show the proton density $n_{p}$, temperature $T_{p}$, temperature anisotropy $T_{\perp, p} / T_{\|, p}$, and parallel plasma beta $\beta_{\|}=v_{t h, \|}^{2} / V_{A}^{2}\left(v_{t h, \|}\right.$ is the proton parallel thermal speed, where the suffix $p$ has been dropped for simplicity), all of which are relatively smooth during the selected interval (between the vertical dotted lines).

The temperature anisotropy $T_{\perp, p} / T_{\|, p}=1.72$ observed at PSP suggests a preferential plasma heating perpendicular to the magnetic field (presumably through dissipation of lowfrequency magnetohydrodynamic turbulence; see Zank et al. 2021, for a comprehensive review of theoretical turbulence models and their convergence with PSP and SO observations). At the observed parallel plasma beta $\beta_{\|}=0.66$ (i.e., the plasma is magnetically dominated), such an anisotropy is above the theoretical threshold for proton cyclotron instability (see, e.g., Hellinger et al. 2006; Telloni et al. 2019), which is therefore at work generating ion-cyclotron waves, as confirmed by windowed Fourier transforms of PSP magnetometer data in the 3-8 $\mathrm{Hz}$ range (not shown).

The plasma stream detected with PSP originated from the equatorial region of the east limb solar corona imaged by Metis $^{47}$ on 2021 January 17 at 16:30 UT simultaneously in polarized brightness (pB) and in H I Ly $\alpha$ ultraviolet (UV) light (Figures 3(a) and (d), respectively). This displays a typical solar minimum configuration with enhanced emission in the equatorial plane.

Two structures can be identified within the region of enhanced emission in both $\mathrm{pB}$ and UV. They are better distinguished by applying a normalizing-radial-graded filter

\footnotetext{
$\overline{47}$ The images were processed and calibrated according to the procedures adopted in Romoli et al. (2021).
}
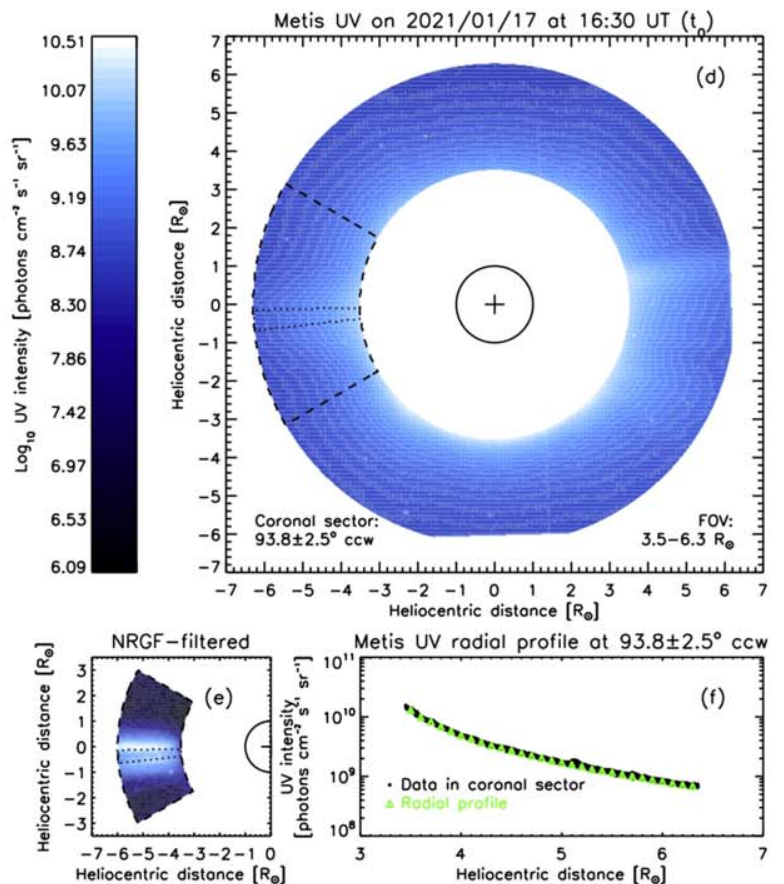

RGF; Morgan et al. 2006) to both images in an angle sector (delimited by dashed lines) $\pm 30^{\circ}$ wide centered on the equator (Figures 3(b) and (e), respectively). The double equatorial structure is easily interpreted in light of the Wilcox Solar Observatory synoptic charts of the coronal magnetic field at the source surface. ${ }^{48}$ In the Metis POS the coronal current sheet was intersecting the equator. However, at about $\pm 30^{\circ}$ in longitude from it were two small sheet warps, with maximum latitude extension to about $\pm 10^{\circ}$, that might explain the double equatorial structure around the equator. This interpretation is also supported by the double HCS crossing experienced by PSP during its approach to the Sun (approximately on 2021 January 17 at 13: 30 UT and on 2021 January 19 at 21:00 UT; see Figure 2(c)).

The solar wind plasma crossing PSP came from the coronal sector \pm 2.5 wide centered at 93.8 , which is the latitude reached by PSP on the POS of Metis during the observation time (Figure 1(a)). This sector is delimited by dotted lines in Figures 3(a), (b), (d), and (e). Black dots in Figures 3(c) and (f) refer to $\mathrm{pB}$ and $\mathrm{UV}$ data in the selected coronal region, while green triangles denote the averaged values in steps of $0.1 R_{\odot}$, from 3.5 to $6.3 R_{\odot}$. The $\mathrm{pB}$ and UV radial profiles are then used to infer the electron density $n_{e}$ and the radial outflow velocity $V_{R}$ of the coronal plasma that is later impinging on the PSP in situ instruments.

Specifically, the electron density is derived by applying the inversion technique developed by van de Hulst (1950) and based on fitting $\mathrm{pB}$ data with a polynomial function (red line in Figure 3(c)). These values are reported in Figure 4(a) (black diamonds) along with a polynomial fit (red line) extrapolated to the PSP location: the very good agreement with the PSP

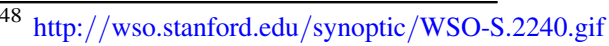



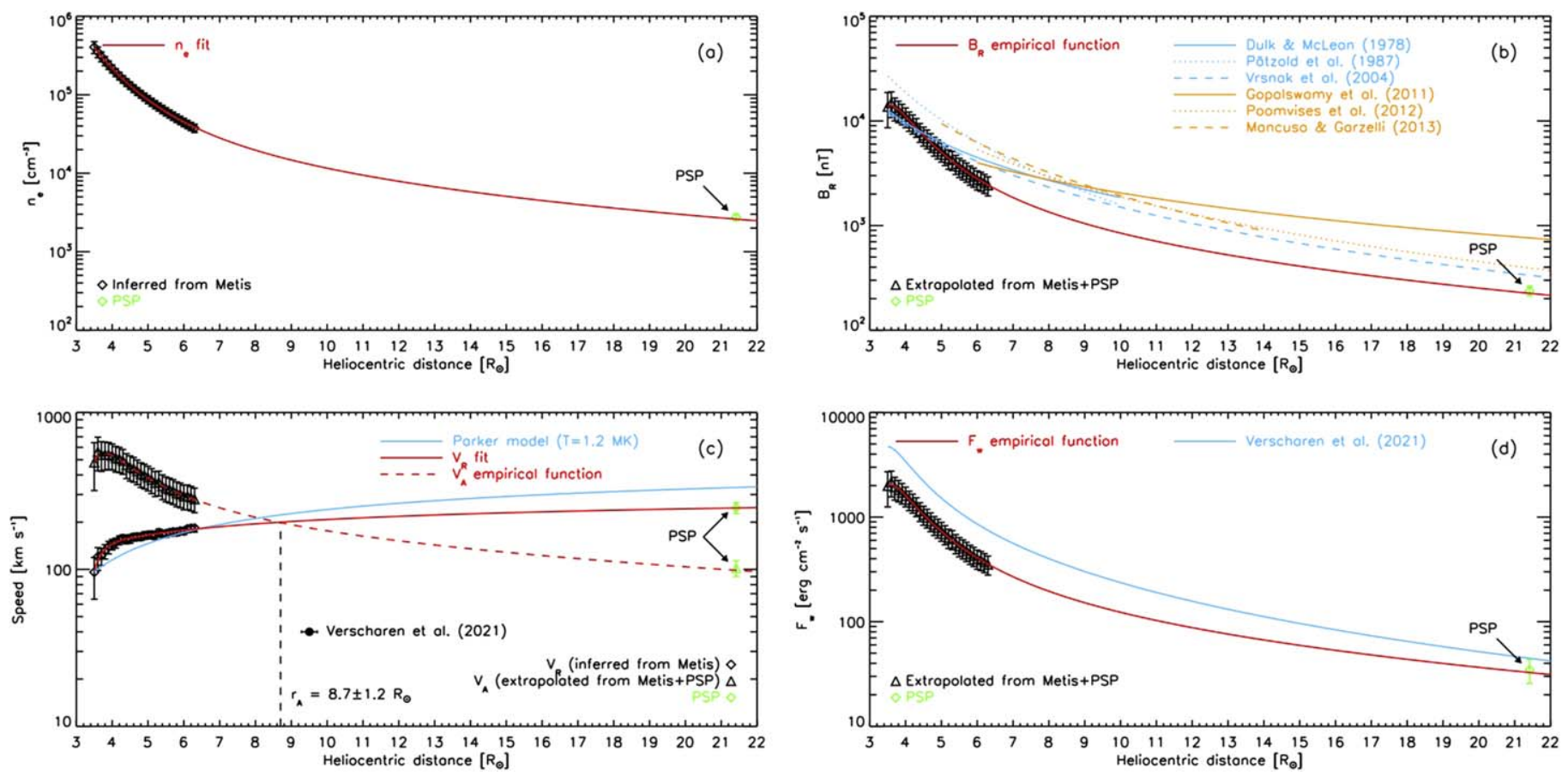

Figure 4. Radial evolution of electron density $n_{e}$ (a), radial component of the magnetic field $B_{R}$ (b), radial coronal outflow $V_{R}$ and Alfvén speed $V_{A}$ (c), and bulk kinetic energy flux density of the solar wind $F_{w}$ (d), at coronal (black signs) and PSP (green sign, pointed by an arrow) heights. Diamonds denote direct Metis/PSP observations, while triangles mark extrapolated values based on conservation laws and PSP data as described in the text. Red solid or dashed curves denote corresponding fit and/or extrapolated empirical functions. The vertical dashed line and the blue solid curve in (c) indicate the inferred Alfvén radius $r_{\mathrm{A}}=8.7 \pm 1.2 R_{\odot}$ (compared with the result by Verscharen et al. 2021, black full circle) and the Parker model for an isothermal corona with $T=1.2 \times 10^{6} \mathrm{~K}$, respectively. As shown in the legend, empirical profiles previously reported in the literature are shown in (b) and (d) for comparison. Uncertainties of the derived quantities are drawn as error bars.

measurement (green diamond: $n_{e, \mathrm{PSP}}=n_{p, \mathrm{PSP}} / 0.95$, assuming a fully ionized plasma with $2.5 \%$ helium, according to Moses et al. 2020) corroborates the fact that the same element of plasma is indeed followed from the coronal region, where it is undergoing acceleration during its outward propagation, to the PSP position in the very inner heliosphere. The radial component of the coronal wind velocity on the POS is obtained by the Doppler dimming technique applied to a 3D solar coronal modeled on the basis of the so-inferred electron density and the measured UV H I Ly $\alpha$ intensity (the reader is referred to Antonucci et al. 2020b; Romoli et al. 2021, for an exhaustive review on how to infer outflow velocities from UV radiative lines observed in the expanding corona and for the Metis first light observations of the coronal solar wind, respectively), under the following assumptions: (i) a hydrogen temperature $T_{p}=1.6 \times 10^{6} \mathrm{~K}$ (Antonucci et al. 2005); (ii) a helium abundance of $2.5 \%$ (Moses et al. 2020); (iii) a temperature anisotropy $T_{\perp, p} / T_{\|, p}=1.72$ as constrained by PSP observations; (iv) the electron temperature derived by Gibson et al. (1999); (v) the chromospheric H I Ly $\alpha$ profile given by Auchère (2005), scaled to the irradiance on 2021 January $17\left(5.42 \times 10^{15}\right.$ photons $\left.\mathrm{cm}^{2} \mathrm{~s}^{-1} \mathrm{sr}^{-1}\right)$, as provided by the LASP Interactive Solar Irradiance Data Center. ${ }^{49}$ The resulting coronal $V_{R}$ values are displayed as black diamonds in Figure 4(c), where they are compared with the Parker outflow solution for an isothermal solar corona with $T=1.2 \times 10^{6} \mathrm{~K}$ (Parker 1958, blue solid line).

The coronal solar wind velocity undergoes a rapid increase with heliocentric distance, from $\sim 80$ to $\sim 150 \mathrm{~km} \mathrm{~s}^{-1}$, in the range from 3.5 to $4.5 R_{\odot}$. Above this height, the acceleration

\footnotetext{
49 https://lasp.colorado.edu/lisird/data/composite_lyman_alpha/
}

slows down and a velocity of $\sim 180 \mathrm{~km} \mathrm{~s}^{-1}$ is reached at 6.3 $R_{\odot}$. Some residual acceleration persists up to the PSP location, where a solar wind speed of $247 \mathrm{~km} \mathrm{~s}^{-1}$ is measured locally (green diamond). Despite the overall good agreement with the purely hydrodynamic Parker model, in order to estimate as accurately as possible the transit time of the plasma volume from the Metis FOV $\left(r_{1}=3.5 R_{\odot}\right)$ to the PSP location $\left(r_{2}=0.1 \mathrm{au}=21.4 R_{\odot}\right)$, Metis and PSP results were fitted to a parametric curve (solid red line: sum of a log-normal distribution and logarithmic quadratic function), which has no interpretative ambition, but aims only at defining an analytical profile. The transit time results in $t=\frac{1}{r_{2}-r_{1}} \int_{r_{1}}^{r_{2}} V(r) d r=16.3 \pm 1.1 \mathrm{hr}$, thus confirming, as mentioned above, that PSP was measuring the same plasma observed with Metis during its propagation across the coronal region.

The simultaneous Metis and PSP observation of the same solar wind plasma is a noticeable step forward allowing the estimation of the coronal magnetic field beyond a few solar radii (not directly measurable in the corona nowadays). This is achieved by applying the conservation of mass and magnetic flux (assuming flux-freezing) to Metis measurements of density and velocity (black diamonds in Figures 4(a) and (c)) and using the plasma and magnetic field data obtained at PSP, according to, e.g., Wang (1995). The so-extrapolated coronal magnetic field along the plasma flow $B_{R}$, in the equatorial region close to the coronal current sheet, is displayed in Figure 4(b) (black triangles): it varies from $\sim 10^{4}$ to $\sim 2 \times 10^{3} \mathrm{nT}$ from 3.5 to 6.3 $R_{\odot}$. These values are somewhat lower than those previously reported in the literature (Dulk \& McLean 1978; Pätzold et al. 1987; Vršnak et al. 2004; Gopalswamy \& Yashiro 2011; 
Poomvises et al. 2012; Mancuso \& Garzelli 2013; shown in Figure 4(b) with different colors and line styles) based solely on remote sensing observations (and thus suffering from LOS integration effects) and/or in situ measurements very far from the Sun (thus not allowing a clear connection to the coronal source regions). In the present case, however, the accuracy of the values inferred for the coronal magnetic field is much higher due to the proximity of PSP, whose local measurements of the same plasma observed with Metis act as a constraint for extrapolations into the extended corona. Similarly, but considering $n_{e}$ and $V_{R}$ fits, an empirical function for $B_{R}$ (red line) can be extrapolated using the aforementioned conservation laws and PSP data.

Given the estimate of the coronal magnetic field, it is then also possible to extrapolate the Alfvén speed $V_{A}$ and, in turn, the Alfvén radius $r_{A}$ along the coronal current sheet, as done for $B_{R}$. Similarly, the solar wind bulk kinetic energy flux density $F_{w}$ can be obtained considering the conservation of total energy along the stream line (see, e.g., Wang 1995 for details). The corresponding empirical trends are also deduced. The extrapolated values for $V_{A}$ and $F_{w}$ are shown as black triangles in Figures 4(c) and (d), respectively. Specifically, $F_{w}$ varies from $\sim 2 \times 10^{3}$ to $\sim 4 \times 10^{2} \mathrm{erg} \mathrm{cm}^{-2} \mathrm{~s}^{-1}$ from 3.5 to $6.3 R_{\odot}$ and is in fair agreement with the empirical profile deduced by Verscharen et al. (2021) on the basis of a two-fluid magnetohydrodynamics framework and Ulysses observations, when neglecting enthalpy, magnetic stresses, and nonradial components of the solar wind flow velocity (blue solid line in Figure 4(d)). Additionally, taking advantage of the $V_{A}$ empirical function (red dashed curve in Figure 4(c), deduced in the same way as that relative to $B_{R}$ ), the Alfvén radius $r_{A}$ can be estimated to be $8.7 \pm 1.2 R_{\odot}$ (in agreement, within uncertainties, with the value of $9.5 \pm 0.2 R_{\odot}$ found by Verscharen et al. 2021). This result is extremely interesting since, during the upcoming SO-PSP quadrature on 2022 June 1, PSP will skim the Metis FOV that will extend from 5.6 to $11.9 R_{\odot}$, thus likely allowing for the very first time the study of the transition of the coronal plasma from a sub-Alfvénic to a super-Alfvénic regime. Interestingly, the initial increase of $B_{R}$ (and, in turn, $V_{A}$ and $F_{w}$ ) up to about $3.8 R_{\odot}$ might depend on residual convergence of the magnetic field lines along the coronal current sheet before starting to diverge (see discussion in, e.g., Schatten et al. 1969; Pinto \& Rouillard 2017).

It is worth noting that the above estimates have been inferred assuming a helium abundance of 2.5\% (Moses et al. 2020). This value can be considered a lower limit (in fact, it refers to a particularly weak solar minimum). For the sake of completeness, it is therefore of interest to report how much the estimates of outflow velocity, magnetic field, kinetic energy flux, and Alfvén point vary assuming an upper limit of helium abundance of, say, $10 \%$. The velocity and kinetic energy flux would be reduced on average by $5.7 \%$, while the average reduction in the magnetic field and Alfvén speed would be $17.6 \%$ and $11.8 \%$, respectively. Finally, the Alfvén radius would approach the Sun by $0.5 R_{\odot}$, i.e., it would be $8.2 R_{\odot}$.

This is the first time that crucial quantities such as the radial magnetic field in the outer corona, the Alfvén radius, and the bulk kinetic energy flux density of the solar wind have been extrapolated in the wind acceleration region with such an accuracy due to the opportunity offered by the first SO-PSP quadrature. Moreover, it is worth noting that, as PSP approaches the solar corona more and more closely and finally enters it (crossing the Alfvén surface), the accuracy in deriving such quantities will further improve, providing a powerful tool to characterize, thanks to Metis observations, the corona in its expansion.

Solar Orbiter is a space mission of international collaboration between ESA and NASA, operated by ESA. D.T. was partially supported by the Italian Space Agency (ASI) under contract 2018-30-HH.0. L.S.V. was funded by the Swedish Contingency Agency, grant 2016-2102, and by SNSA, grant86/20. L. D.W. is supported by the STFC consolidated grant ST/ S000364/1. G.P.Z. acknowledges the partial support of a NASA PSP contract SV4-84017, an NSF EPSCoR RII-Track-1 Cooperative Agreement OIA-1655280, and a NASA IMAP grant through SUB000313/80GSFC19C0027. O.P. was supported by the NASA grant 80NSSC 20K1829. L.S. was supported by a grant from the NASA Heliophysics Technology and Instrument Development for Science Program, NNH15ZDA001N-HTIDS, and also by the basic Research Funds of the Office of Naval Research. D.V. is supported by STFC Ernest Rutherford Fellowship ST/P003826/1 and STFC Consolidated Grant ST/S000240/1. The Metis program is supported by ASI under contracts to the National Institute for Astrophysics and industrial partners. Metis was built with hardware contributions from Germany (Bundesministerium für Wirtschaft und Energie through the Deutsches Zentrum für Luft- und Raumfahrt e.V.), the Czech Republic (PRODEX) and ESA. The FIELDS and SWEAP teams acknowledge support from NASA contract NNN06AA01C. PSP data were downloaded from the NASA's Space Physics Data Facility (https:// spdf.gsfc.nasa.gov). D.T. wishes to thank Masaru Nakanotani for his help in sketching the cartoon of Figure 1.

\section{ORCID iDs}

Daniele Telloni (ib https://orcid.org/0000-0002-6710-8142 Vincenzo Andretta (1D https://orcid.org/0000-0003-1962-9741 Ester Antonucci (1) https://orcid.org/0000-0003-4155-6542 Alessandro Bemporad (1) https://orcid.org/0000-00015796-5653

Giuseppe E. Capuano (10) https://orcid.org/0000-00028430-8218

Silvano Fineschi (D) https://orcid.org/0000-0002-2789-816X Silvio Giordano (i) https://orcid.org/0000-0002-3468-8566 Shadia Habbal (i) https://orcid.org/0000-0003-4089-9316 Denise Perrone (iD https://orcid.org/0000-0003-1059-4853 Rui F. Pinto (1) https://orcid.org/0000-0001-8247-7168 Luca Sorriso-Valvo (i) https://orcid.org/0000-0002-5981-7758 Daniele Spadaro (iD https://orcid.org/0000-0003-3517-8688 Roberto Susino (iD https://orcid.org/0000-0002-1017-7163 Lloyd D. Woodham (iD https://orcid.org/0000-00032845-4250

Gary P. Zank (iD https://orcid.org/0000-0002-4642-6192 Marco Romoli (i) https://orcid.org/0000-0001-9921-1198 Stuart D. Bale (i) https://orcid.org/0000-0002-1989-3596 Justin C. Kasper (i) https://orcid.org/0000-0002-7077-930X Frédéric Auchère (i) https://orcid.org/0000-0003-0972-7022 Roberto Bruno (i) https://orcid.org/0000-0002-2152-0115 Gerardo Capobianco (10 https://orcid.org/0000-00030520-2528

Anthony W. Case (iD https://orcid.org/0000-0002-3520-4041 Chiara Casini (i) https://orcid.org/0000-0001-8783-0047 Marta Casti (i) https://orcid.org/0000-0002-9716-3820 
Paolo Chioetto (iD https://orcid.org/0000-0002-3379-2142

Alain J. Corso (iD https://orcid.org/0000-0003-0378-9249

Vania Da Deppo (i) https://orcid.org/0000-0001-6273-8738

Yara De Leo (1) https://orcid.org/0000-0003-2426-2112

Thierry Dudok de Wit (1) https://orcid.org/0000-00024401-0943

Federica Frassati (ib https://orcid.org/0000-0001-9014-614X

Fabio Frassetto (iD https://orcid.org/0000-0001-5528-1995

Keith Goetz (iD https://orcid.org/0000-0003-0420-3633

Salvo L. Guglielmino (ib https://orcid.org/0000-0002-

1837-2262

Peter R. Harvey (1D https://orcid.org/0000-0002-6938-0166

Petr Heinzel (i) https://orcid.org/0000-0002-5778-2600

Giovanna Jerse (iD https://orcid.org/0000-0002-0764-7929

Kelly E. Korreck (iD https://orcid.org/0000-0001-6095-2490

Federico Landini (iD https://orcid.org/0000-0001-8244-9749

Davin Larson (1D https://orcid.org/0000-0001-5030-6030

Alessandro Liberatore (iD https://orcid.org/0000-0002-

0016-7594

Roberto Livi (iD https://orcid.org/0000-0002-0396-0547

Robert J. MacDowall (10 https://orcid.org/0000-0003-

3112-4201

Enrico Magli (1) https://orcid.org/0000-0002-0901-0251

David M. Malaspina (I) https://orcid.org/0000-0003-

1191-1558

Giuseppe Massone (ib https://orcid.org/0000-0002-2656-1557

Mauro Messerotti (i) https://orcid.org/0000-0002-5422-1963

John D. Moses (iD https://orcid.org/0000-0001-9670-2063

Giampiero Naletto (i) https://orcid.org/0000-0003-2007-3138

Gianalfredo Nicolini (iD https:// orcid.org/0000-0002-

9459-3841

Giuseppe Nisticò (iD https://orcid.org/0000-0003-2566-2820

Olga Panasenco (i) https://orcid.org/0000-0002-4440-7166

Maurizio Pancrazzi (10 https://orcid.org/0000-0002-3789-2482

Maria G. Pelizzo (iD https://orcid.org/0000-0002-1383-6750

Marc Pulupa (iD https://orcid.org/0000-0002-1573-7457

Fabio Reale (1) https://orcid.org/0000-0002-1820-4824

Paolo Romano (1) https://orcid.org/0000-0001-7066-6674

Clementina Sasso (iD https://orcid.org/0000-0002-5163-5837

Udo Schühle (iD https://orcid.org/0000-0001-6060-9078

Marco Stangalini (D) https://orcid.org/0000-0002-5365-7546

Michael L. Stevens (i) https://orcid.org/0000-0002-7728-0085

Leonard Strachan (i) https://orcid.org/0000-0002-5425-7122

Thomas Straus (i) https://orcid.org/0000-0002-6280-806X

Luca Teriaca (ib https://orcid.org/0000-0001-7298-2320

Michela Uslenghi (iD https://orcid.org/0000-0002-7585-8605

Marco Velli (i) https://orcid.org/0000-0002-2381-3106

Daniel Verscharen (iD https://orcid.org/0000-0002-0497-1096
Cosimo A. Volpicelli (iD https://orcid.org/0000-00024997-1460

Phyllis Whittlesey (iD https://orcid.org/0000-0002-7287-5098

Luca Zangrilli (i) https://orcid.org/0000-0002-4184-2031

Gaetano Zimbardo (iD https://orcid.org/0000-0002-9207-2647

Paola Zuppella (iD https://orcid.org/0000-0003-0290-3193

\section{References}

Antonucci, E., Abbo, L., \& Dodero, M. A. 2005, A\&A, 435, 699 Antonucci, E., Harra, L., Susino, R., \& Telloni, D. 2020a, SSRv, 216, 117 Antonucci, E., Romoli, M., Andretta, V., et al. 2020b, A\&A, 642, A10 Auchère, F. 2005, ApJ, 622, 737

Bale, S. D., Goetz, K., Harvey, P. R., et al. 2016, SSRv, 204, 49

Bale, S. D., Badman, S. T., Bonnell, J. W., et al. 2019, Natur, 576, 237

Bemporad, A., Poletto, G., Suess, S. T., et al. 2003, ApJ, 593, 1146

Bradshaw, S. J., \& Raymond, J. 2013, SSRv, 178, 271

Brueckner, G. E., Howard, R. A., Koomen, M. J., et al. 1995, SoPh, 162, 357

Bruno, R., \& Carbone, V. 2013, LRSP, 10, 2

Dolei, S., Susino, R., Sasso, C., et al. 2018, A\&A, 612, A84

Domingo, V., Fleck, B., \& Poland, A. I. 1995, SoPh, 162, 1

Dulk, G. A., \& McLean, D. J. 1978, SoPh, 57, 279

Fox, N. J., Velli, M., Bale, S. D., et al. 2016, SSRv, 204, 7

Gibson, S. E., Fludra, A., Bagenal, F., et al. 1999, JGRA, 104, 9691

Gloeckler, G., Geiss, J., Balsiger, H., et al. 1992, A\&AS, 92, 267

Gopalswamy, N., \& Yashiro, S. 2011, ApJL, 736, L17

Hellinger, P., Trávníček, P., Kasper, J. C., \& Lazarus, A. J. 2006, GeoRL, 33, L09101

Kasper, J. C., Abiad, R., Austin, G., et al. 2016, SSRv, 204, 131

Kohl, J. L., Esser, R., Gardner, L. D., et al. 1995, SoPh, 162, 313

Mancuso, S., \& Garzelli, M. V. 2013, A\&A, 553, A100

Morgan, H., Habbal, S. R., \& Woo, R. 2006, SoPh, 236, 263

Moses, J. D., Antonucci, E., Newmark, J., et al. 2020, NatAs, 4, 1134

Müller, D., St., Cyr, O. C., Zouganelis, I., et al. 2020, A\&A, 642, A1

Parker, E. N. 1958, ApJ, 128, 664

Pätzold, M., Bird, M. K., Volland, H., et al. 1987, SoPh, 109, 91

Pinto, R. F., \& Rouillard, A. P. 2017, ApJ, 838, 89

Poletto, G., Suess, S. T., Biesecker, D. A., et al. 2002, JGRA, 107, 1300

Poomvises, W., Gopalswamy, N., Yashiro, S., Kwon, R.-Y., \& Olmedo, O. 2012, ApJ, 758, 118

Romoli, M., Antonucci, E., Andretta, V., et al. 2021, A\&A, in press

Schatten, K. H., Wilcox, J. M., \& Ness, N. F. 1969, SoPh, 6, 442

Suess, S. T., Poletto, G., Romoli, M., et al. 2000, JGRA, 105, 25033

Suess, S., \& Poletto, G. 2001, SSRv, 97, 59

Telloni, D., Carbone, F., Bruno, R., et al. 2019, ApJL, 885, L5

Telloni, D., Sorriso-Valvo, L., Woodham, L. D., et al. 2021, ApJL, 912, L21 van de Hulst, H. C. 1950, BAN, 11, 135

Velli, M., Harra, L. K., Vourlidas, A., et al. 2020, A\&A, 642, A4

Verscharen, D., Klein, K. G., \& Maruca, B. A. 2019, LRSP, 16, 5

Verscharen, D., Bale, S. D., \& Velli, M. 2021, MNRAS, 506, 4993

Vršnak, B., Magdalenić, J., \& Zlobec, P. 2004, A\&A, 413, 753

Wang, Y.-M. 1995, ApJL, 449, L157

Wenzel, K. P., Marsden, R. G., Page, D. E., \& Smith, E. J. 1992, A\&AS, 92, 207

Woodham, L. D., Horbury, T. S., Matteini, L., et al. 2021, A\&A, 650, L1

Zank, G. P., Zhao, L.-L., Adhikari, L., et al. 2021, PhPl, 28, 080501

Zouganelis, I., De Groof, A., Walsh, A. P., et al. 2020, A\&A, 642, A3 